\title{
Practice does not facilitate acquisition of McCollough effects: Evidence against a learning model
}

\author{
D. SKOWBO and J. RICH \\ Colby College, Waterville, Maine
}

\begin{abstract}
Previous investigators (Schmidt, Pinette, \& Finke, 1978) have asserted that McCollough effects (MEs) are acquired more readily by experienced than by inexperienced subjects. The present experiments examine this claim in a paradigm that utilizes quantitative measurements of MEs to evaluate possible changes in subjects' susceptibility to them. MEs were induced every few days for approximately 2 monthe; results revealed no progressive increments in either strength or acquisition rate. The lack of facilitation due to practice is inconsistent with learning models proposed to account for MEs.
\end{abstract}

Aftereffects of color that are contingent upon the orientation of lines were first described by McCollough (1965) and now are commonly referred to as McCollough effects (MEs) or as contingent aftereffects. After a subject has inspected chromatic grating patterns, he may perceive achromatic gratings as being tinted with a hue approximately complementary to that presented during the inspection period. MEs have spatial characteristics that resemble those of visual feature detectors, and some attempts to explain MEs have been based on adaptation in channels sensitive to color and form (see, for example, the reviews by Eimas \& Miller, 1978, and Stromeyer, 1978). Other investigators have argued that MEs resemble classically conditioned responses. A leading proponent of this viewpoint is Murch (1976), who suggested that the appearance of color is the product of a visual response that becomes associated with and consequently evoked by the presentation of contours in a particular spatial configuration.

Schmidt, Pinette, and Finke (1978) presented arguments that a response change occurring through adaptation differs from one occurring through learning in that the former process generally is not affected by repetition or experience. A behavior modified only by adaptation would presumably show the same characteristics no matter how many times that adaptation had taken place (Kling, 1971). Schmidt et al. offered their impression from previous work that experienced subjects acquire

The authors are grateful for Keith White's helpful comments on an earlier version of this paper. We thank $H$. MacDuffie for assistance with data collection, and also T. Forster, J. Gladstone, C. Goulston, B. Leonard, M. Lingafelter, J. Peterson, and M. Thurston for serving as subjects. This research was supported by grants from Colby College. Requests for reprints should be sent to D. Skowbo, Psychology Department, Colby College, Waterville, Maine 04901. J. Rich is now at New School for Social Research, New York, New York 10011.
MEs more readily than do inexperienced subjects, a notion consistent with the view that. MEs are better described as the result of learning than of simple adaptation. As Schmidt et al. stated, "If indeed the effects are more easily acquired with practice, the strong implication is that the associations between patterns and colors are the results of a learning process" (1978, p. 126).

Schmidt et al. then performed an experiment to demonstrate that successively fewer exposures to the inducing stimuli were required to establish MEs to criterion reliability. Their subjects underwent trials consisting of two presentations each of two chromatic patterns, followed by a viewing of four test patterns. Repeated trials continued until the subject satisfied a criterion of appropriate indications as to which portions of all four test patterns appeared redder. In subsequent phases of the experiment, the effect was repeatedly reversed, that is, reestablished to criterion with color-pattern combinations opposite to those used in the immediately preceding acquisition phase. Subjects acquired the reversed effects with fewer adaptation trials at the end of an eight-reversals series than at the beginning, and Schmidt et al. took this to be evidence of conditioning processes in MEs.

We question the appropriateness of using a shortterm experiment to test the hypothesis that MEs are more readily acquired with experience; thus, we are unsure whether Schmidt et al.'s results do in fact support a learning model of MEs. For example, when three of the four subjects had already participated in previous ME studies, we wonder whether it is logical to attribute variations observed within a single study to changes in subjects' susceptibility to MEs.

We suggest that a confounding variable may be present in Schmidt et al.'s design. White (1977) showed that immediate reversals of MEs would produce not a series of new, independent effects, but rather net MEs that represent the combination of prior effects in various 
stages of decay. Thus, in Schmidt et al.'s study, it would be difficult to separate the expected results of combining MEs from the hypothesized changes in subjects' susceptibility to successive inductions. Consistent with White's (1977) predictions and findings, however, Schmidt et al.'s subjects did report that later reversals produced weaker effects than earlier ones.

In addition to the issues raised above regarding interpretation of results, we are concerned with the problems of operationally defining "susceptibility" to MEs. A practiced subject who reports the presence of MEs with more consistency and certainty than a naive one (another observation reported by Schmidt et al., 1978) may simply have acquired an increased familiarity with the phenomenon and/or an increased willingness to report a perception of weakly saturated colors. In order to assess changes in vulnerability to MEs, judgments about the effects should be as free as possible from expectation, demand characteristics of testing, etc.; we feel that the use of a quantitative measure of ME strength would help to remove these sources of ambiguity.

Given such a measure, increased susceptibility might mean that (1) with constant periods of adaptation, the measure indicates stronger effects with increasing numbers of inductions, or (2) the rate of acquisition is more rapid with increasing numbers of inductions. Possibility 2 seems consistent with Shute's (1979) observation that "almost all workers who habitually induce in themselves McCollough effects find that increasingly short adaptation times are required to do so" (p. 18). The second possibility would reveal itself as progressively fewer exposures to induction stimuli being required to reach some criterion strength (as in the Schmidt et al. paradigm), or else as progressively steeper acquisition curves if the development of the effect were tracked with repeated tests over the course of the acquisition periods.

The present experiments were designed to eliminate the questions we have raised concerning Schmidt et al.'s study and thus to adequately test the hypothesis that acquisition characteristics of MEs are altered by repeated inductions. Using a quantifiable index of ME strength, we looked for long-term changes in the two characteristics described in the preceding paragraph-strength at the end of successive acquisition periods (Experiment 1), and rate of growth within successive acquisition periods (Experiment 2).

\section{EXPERIMENT 1}

\section{Method}

Subjects. There were six subjects: three males and three females. All had color-discriminating ability in the superior range, as measured by the Farnsworth-Munsell 100 Hue Test, and all were given extensive training in matching weakly saturated stimuli with the color-mixing device described below. Two had seen an ME once as part of a class demonstration several months before the experiment began; the others had had no prior exposure at all to MEs. None were aware of the hypothesis being tested and, in fact, were told that the experimenter was collecting descriptive data.
Apparatus. During the sessions, the subjects sat facing two adjacent circular fields $11 \mathrm{deg}$ in diameter on a large black background. Grating patterns used to induce and test the effect were projected onto the left of these fields. The inducing patterns were vertical or horizontal green (Wratten No. 53) or magenta (Wratten No. 32) gratings; space-average luminances were, respectively, 96 and $75 \mathrm{~cd} / \mathrm{m}^{2}$. The test pattern contained vertical lines on its upper half and horizontal lines on the bottom; its space-average luminance was $5 \mathrm{~cd} / \mathrm{m}^{2}$. The spatial frequency of the grating patterns was 1,2 , or 4 cycles/deg; two subjects were assigned to each frequency.

A homogeneous field of variable chromaticity appeared on the right-hand field; its luminance was $7 \mathrm{~cd} / \mathrm{m}^{2}$. Subjects adjusted this stimulus to match effects seen on the adjacent test pattern. The source of this field was a projection colorimeter that mixed light transmitted by two Wratten filters (CC $30 \mathrm{M}$ and CC50G). All mixtures could be located in C.I.E. space along a straight line connecting two points with the coordinates $x=.407$, $\mathrm{y}=.454$ and $\mathrm{x}=.421, \mathrm{y}=.350$.

Procedure. The subjects began each session by adapting for $3 \mathrm{~min}$ to the homogeneous field set at a previously established neutral point. Then the test pattern appeared on the left field and the subjects made six matches, in ABBA sequence, to each of the two portions of the pattern. MEs were induced subsequently by having subjects view the chromatic stimuli described above, alternating every $10 \mathrm{sec}$ for a total of $5 \mathrm{~min}$. After another $3 \mathrm{~min}$ of light adaptation, the subjects again gave a series of matches to the test pattern. Each subject participated in 16 sessions, with $3-4$ days elapsing between each session. Preadaptation matches to the test pattern were used to assure that the subjects began no session with residual effects from the previous one. The color-orientation combination used to induce MEs was reversed in successive sessions.

\section{Results}

The means of matches to horizontal and vertical portions of the test pattern were converted to their C.I.E. coordinates. A simple index of strength of MEs obtained in each session was taken to be the distance in C.I.E. space between the coordinates for the two means. ${ }^{1}$

Overall, no reliable trends toward increasing strength over successive induction sessions were evident in the data. One subject did have a tendency to show stronger effects over the course of the experiment; however, another showed a weakening trend of comparable magnitude. The remainder showed no progressive changes. Figure 1 shows the strength index plotted against successive sessions for two sample subjects. As is evident in this figure, intersession variability characterized the data.

Figure 2 shows a highly condensed version of results obtained from all subjects. In the main part of the figure, sessions have been divided into blocks (Sessions 1-4, $5-8,9-12,13-16$ ), and data from the two subjects assigned to each spatial frequency have been combined. Thus, this graph shows the strength index plotted against four trial blocks; the different curves represent the spatial frequencies of the grating patterns. Differences in the absolute heights of these curves show the effect of spatial frequency first reported by Stromeyer (1972), but the functions show no consistent or strong changes associated with successive session blocks. The inset at the right of Figure 2 shows data collapsed over all subjects/spatial frequencies. It seems evident from inspection of this graph that there is no main effect due to successive session blocks. 


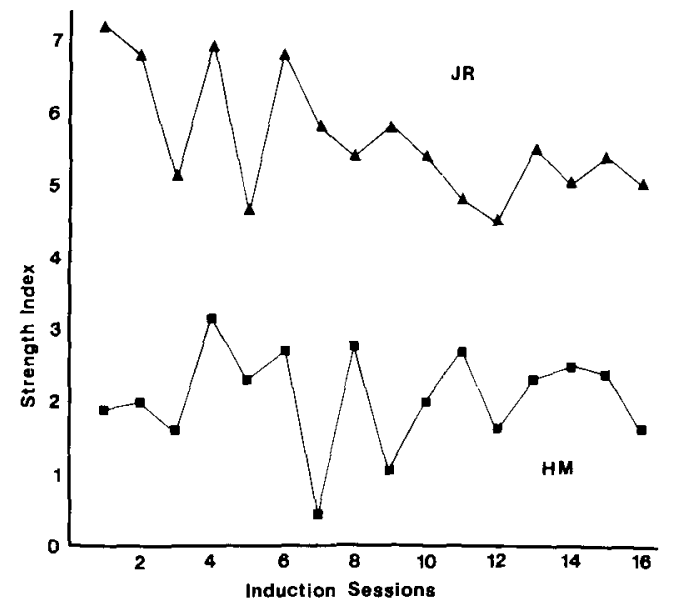

Figure 1. Strength index as a function of successive induction sessions. Numbers on the ordinate are distances in C.I.E. space $\times 10^{2}$. Results from two subjects are shown. Spatial frequency of the gratings was 4 cycles/deg for J.R. and 1 cycle/deg for H.M.

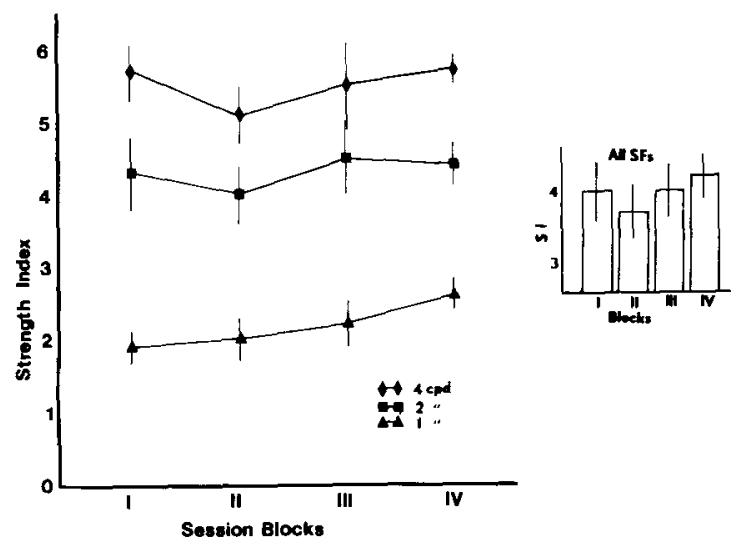

Figure 2. Strength index as a function of session blocks. Numbers on the ordinate are distances in C.I.E. space $\times 10^{2}$. On the abscisa, Block I includes Sessions 1-4; Block II is Sessions 5-8; Block III is 9-12; and Block IV is 13-16. In the main graph, different symbols represent the three spatial frequencies. Each symbol shows the mean of eight strength assessments: four sessions for each of the two subjects assigned a particular spatial frequency. Vertical lines show \pm 1 estimated standard error from the means. In the inset to the right, data from all subjects have been combined. The height of the bars shows the mean of 24 assessments: four sessions for each of six subjects. Again, the vertical lines show \pm 1 estimated standard error from these means.

\section{EXPERIMENT 2}

\section{Method}

Subjects. Three subjects, one male and two females, were used. None had had any prior exposure at all to MEs. They were similar to the subjects in Experiment 1 in all other respects described in the previous Method section.

Apparatus. The apparatus was identical to that described for Experiment 1 . However, only the 2-cycle/deg patterns were used.

Procedure. After an initial lightadaptation period lasting $3 \mathrm{~min}$, the subjects gave one match to each portion of the test pattern. They subsequently viewed a green or magenta vertical grating for $5 \mathrm{sec}$ and then a horizontal grating of the other color for $5 \mathrm{sec}$. The test pattern reappeared after 2-3 sec of darkness, and the subjects again gave one match for each half of the test field. ${ }^{2}$ Approximately $30-40 \mathrm{sec}$ was required to complete the two matches. The sequence of $10 \mathrm{sec}$ of chromatic adaptation followed by test was repeated until the subjects had made a total of 20 postadaptation measurement pairs. Each subject participated in 15 sessions. As in Experiment 1, the colororientation combination was reversed in successive sessions, 3-4 days elapsed between sessions, and the subjects did not have measurable residual effects from session to session.

\section{Results}

Each match was converted to C.I.E. coordinates, and the distance in C.I.E. space between the matches was taken as an index of ME strength. Plots of the strength index against the 20 measurements taken per session revealed increases in the index with succeeding measurements within each session, but no changes in the rate of growth over successive sessions were evident. Figure 3 shows the data from Experiment 2 in a highly condensed form. Strength index against measurements within sessions is shown with the data collapsed over all subjects and with the different curves representing data obtained in Sessions 1-5, 6-10, and 11-15. These three grouped curves are indistinguishable, as were the individual acquisition curves.

\section{DISCUSSION}

We have been unable to find evidence in support of reports made by Schmidt et al. (1978) and Shute (1979) concerning the influence of practice on MEs. Over a period of weeks, our initially naive subjects showed no change in either the strength or the acquisition rate of successively acquired MEs. We believe that, with increasing numbers of exposures to MEs, subjects do become more adept at recognizing the presence of an often subtle coloration on test patterns. Familiarity, expectation, and responses to demand characteristics of testing might all increase with experience; we feel that "learning

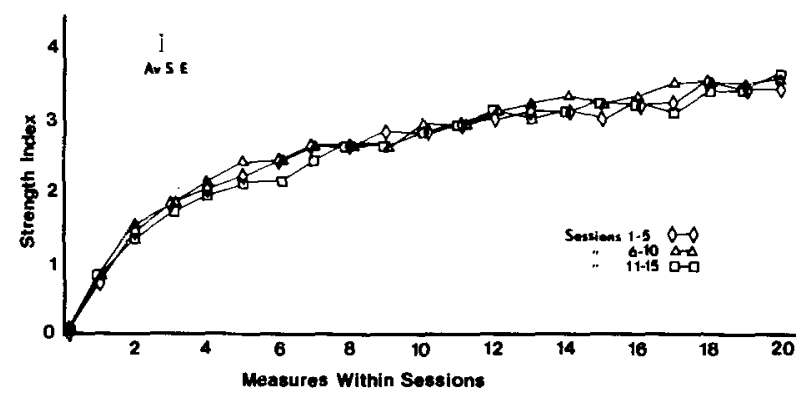

Figure 3. Strength index as a function of assessments made within induction sessions. Data have been collapsed over subjects. Numbers on the ordinate are distances in C.I.E. space $\times 10^{2}$. Different symbols represent three blocks of sessions: 1-5, 6-10, and 11-15. Each symbol shows the mean of 15 assessments: five sessions for each of three subjects. The vertical line in the upper left corner shows the average of estimated standard errors calculated for each set of 15 assessments. 
to report the effect" is a more appropriate description of such processes than "learning to acquire the effect" (Schmidt et al., 1978, p. 126). Shute (1979) has commented, however, that even experienced subjects are often erroneous in their subjective estimates of $\mathrm{ME}$ strength. We concur: Several of our subjects, when debriefed at the conclusion of the experiments, expressed confidence that the hypothesized facilitation effects had occurred.

We interpret the present findings as a challenge to any models that assume that MEs are learned responses. Previous attempts to evaluate learning models have typically described the characteristics of MEs that either do or do not resemble those of classically conditioned responses. Occasionally, the closeness of the resemblance has been debated (see, for example, McCarter \& Silver, 1977, and Murch, 1977). In contrast, the present experiments were based on a fundamental distinction between behavioral changes effected by a learning process and behavioral changes concomitant to such other experiential processes as sensory adaptation. The learning process (whether reflecting classical or operant conditioning, imitation, or some other category) is defined to be influenced by practice (Kling, 1971). We find no such influences within the limits of measurement error; thus, it must be questioned whether any model postulating learning will provide a parsimonious account for MEs.

\section{RTRTRENCAS}

Ermas, P., Minusen, J. Effects of selective adaptation on the perception of speech and visual patterns: Evidence for feature detectors. In R. Walk \& H. Pick (Bds.), Perception and Experience. New York: Plenum Press, 1978.

KIING, J. Learning: Introductory survey. In J. Kling \& L. Riges (Eds.), Woodworth and Schlasberg's experimental psycholozy (3rd ed.). New York: Holt, Rinehart a Winston, 1971.
McCarter, A., Silven, A. The McCollough effect: A classical conditioning phenomenon? Vision Research, 1977, 17, 317-319.

McCollough, C. Color adaptation of edge detectors in the human visual system. Science, 1965, 149, 1115-1116.

Murch, G. Classical conditioning of the McCollough effect: Temporal parameters. Vision Research, 1976, 16, 615-619.

Munch, G. A reply to McCarter and Silver. Vision Research, 1977, 17, 321-322.

Schmidt, M., Pinetre, P., \& Finks, R. Further evidence for conditioning processes in the McCollough effect. Journal of General Psychology, 1978, 99, 117-132.

Shute, C. The McCollough effect: An indicator of central neurotransmitter activity. Cambridge: Cambridge University Press, 1979.

Stwomerer, C. Edge-contingent color aftereffects: Spatial frequency speciflcity. Vision Research, 1972, 12, 717-732.

STmomeren, C. Form-color after effects in human vision. In R. Held, H. Leibowitz, H. L. Teuber (Eds.), Perception (Vol. 8): Handbook of sensory physiology. New York: SpringerVerlag, 1978.

WHITs, K. Summation of successively established orientation contingent color aftereffects. Perception \& Psychophysics, 1977, 22, 123-136.

\section{NOTES}

1. The distance beween loci for an achromatic neutral point (e.g., a preadaptation match to the test pattern) and one aftereffect color is a close approximation of excitation purity for that color. Our strength index, therefore, would approximate an additive combination of the excitation purities for both aftereffect colors.

2. Since the objective in Experiment 2 was to track the growth of the effect within acquisition phases, it was considered undesirable to interrupt the acquisition process for the length of time required to obtain a series of matches each time the effect was assessed. Therefore, in this experiment, each measurement of the effect was accomplished with only one match for each half of the test pattern.

(Manuscript received May 13, 1982; accepted for publication September 21, 1982.) 